\title{
Pigment fallout and uveitis after cryotherapy
}

\author{
A. H. GHIGNELL, R. S. GLEMETT, and I. H. S. REVIE \\ Moorfields Eye Hospital, City Road, London, E.C.I
}

Cryotherapy, first advocated by Bietti (1933), has been widely accepted as a useful means of achieving adhesion in the treatment of retinal detachment. It was later popularised by Lincoff, McLean, and Nano (1964), who subsequently used this form of treatment in their modifications of the Custodis procedure for the treatment of retinal detachment (Lincoff, Baras, and McLean, I965).

Various complications of cryosurgery after its use in the treatment of retinal detachments have been described. Pigment fallout was observed by Abraham and Shea (1968), Johnston and Okun (1969), and Sudarsky and Yannuzzi (1970). Uveitis in the postoperative period was described by Lincoff and McLean (1966) and Abraham and Shea (1968).

The purpose of this study was to observe the incidence and clinical features of pigment fallout and postoperative uveitis, and to study the relationship of these complications to the severity of cryoapplication.

\section{Materials and methods}

I6o consecutive patients admitted to the Retinal Unit at Moorfields Eye Hospital, City Road, for retinal detachment surgery were studied. Those receiving prophylactic treatment only were not considered. In all cases cryotherapy was applied through full-thickness sclera.

The fundus was examined by direct and indirect ophthalmoscopy, scleral depression, and 3mirror gonioscopy. The details were recorded with retinal drawings and in certain cases with paintings and photography. The depth of subretinal fluid at the site of the retinal holes and therefore the intended site of cryotherapy was expressed as balloon, moderate, or slight. The amount of retina to be treated with cryotherapy was expressed as more or less than half a quadrant. Any abnormal preoperative pigmentation of the retina was noted (e.g. pigmented lattice degeneration).

In all cases the Amoils or Amoilette cryotherapy apparatus was used at the time of surgery and cryotherapy was applied before drainage of subretinal fluid and tightening of the buckle sutures. The retina, and in particular the area treated with cryotherapy, was examined with indirect ophthalmoscopy and slit-lamp illumination postoperatively every second day until discharge from hospital Io to 14 days after surgery. Patients with complications were followed until a final assessment of the treated area was made 2 months after surgery.

An additional group of fifty patients in whom no pigment fallout or postoperative uveitis had occurred, was studied 2 months after retinal detachment surgery.

Lincoff and McLean ( 1969 ) showed that the degree of pigmentation of a lesion in rabbits after cryotherapy was proportional to the intensity of application. Clinical examination of the human retina after cryotherapy shows comparable changes, a mild application leading to evenly distributed pigmentation of the treated area and a heavy application leading to a highly atrophic lesion, with clumped pigmentation at the edges. The lesion is white because of destruction of most of the choroidal bloodvessels, only the large vessels being left intact. 
The varying appearance of the treated area has been classified to assess the degree of cryotherapy application (Fig. I). All lesions were classified 2 months after surgery (Chignell, Revie, and Clemett, 197I).
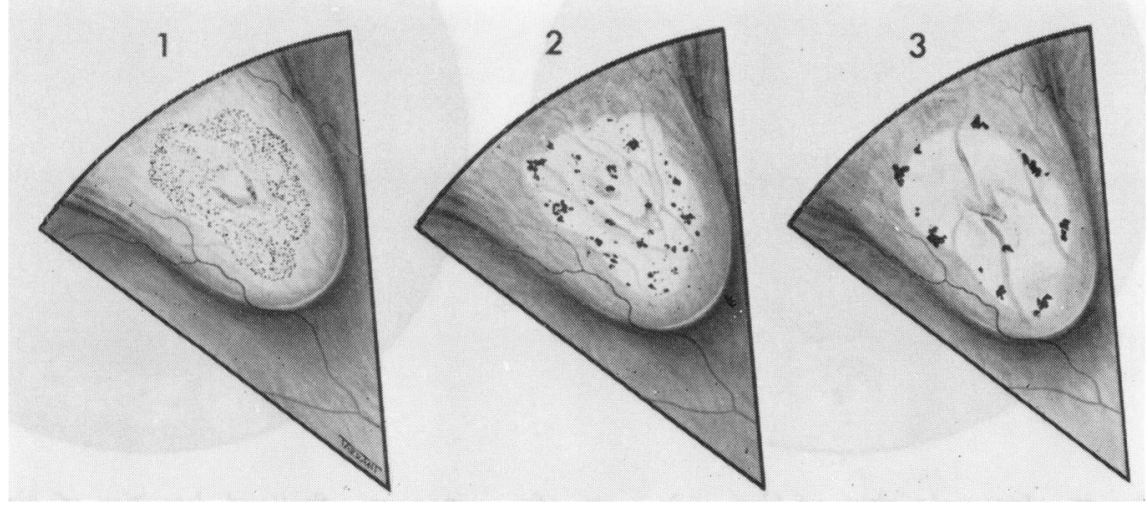

FIG. I Three grades of cryolesion 2 months after surgery

Grade I Even pigmentation of the lesion in a pepper-and-salt pattern.

Grade II Scattered irregular pigmentation with a paler lesion.

Grade III White atrophic scar with variable pigment clumping at the edge of the lesion.

A mixture of grades may be found in any one treated area. There is no evidence to suggest that the production of a Grade III lesion is necessary to achieve good adhesion.

POSTOPERATIVE UVEITIS

Patients who had been treated with surgery involving either drainage of subretinal fluid or encirclement were not considered. In these cases interpretation of physical signs, such as flare and cells in the anterior chamber and vitreous haze, is difficult in the postoperative period. These signs may be produced to a lesser or greater extent by varying degrees of anterior segment ischaemia (Crock, 1967) after encirclement procedures, or by complications after drainage of subretinal fluid (e.g. hypotony, choroidal haemorrhage, and bacterial endophthalmitis).

\section{Results}

PIGMENT FALLOUT

Nineteen patients ( 12 per cent. of the I6o studied) showed evidence of pigment fallout. In all cases pigment fallout occurred by spread within the subretinal fluid. Although spread to the vitreous occurs via the retinal holes (Sudarsky and Yannuzzi, 1970), no cases of pigment fallout due to the precipitation of pigment from the vitreous on previously undetached retina were seen in this study. The fallout of pigment was either linear (Fig. 2) because of gravitational deposition in the supine eye, and corresponding to the posterior limit of the detachment, or occurred in a more generalized way (Fig. 3).

The pigment was either fine or coarse, but mixed appearances were seen. Preoperative retinal pigmentation was assessed in these nineteen cases and was found to be present in eight of them. Two showed pigmented lattice degeneration, five pigmented cobblestone degeneration, and one marked high-water marking from a long-standing retinal detachment. 


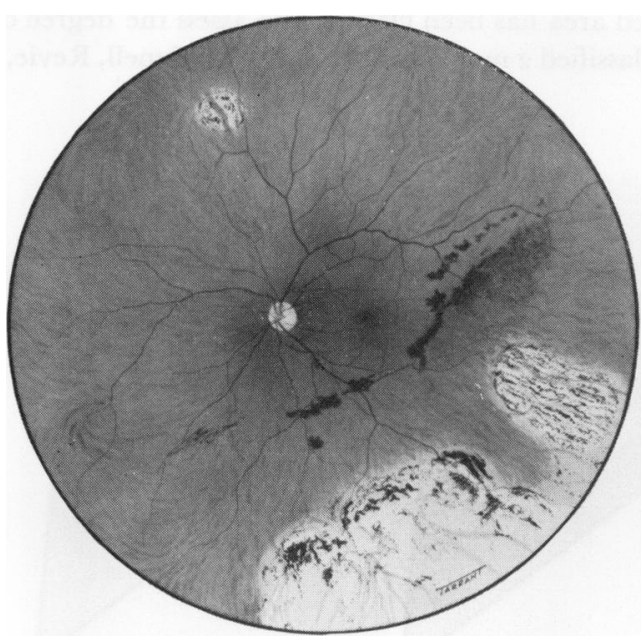

FIG. 2 Linear deposition of pigment in a case of pigment fallout. Excessively heavy cryo-lesion below

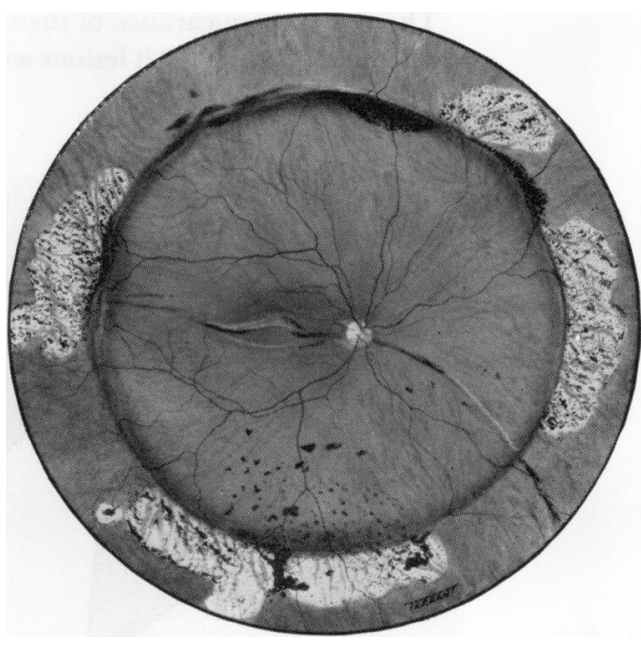

FIG. 3 Scattered deposition of pigment via subretinal fluid in a case of pigment fallout. Highwater mark also seen
Table I Appearance of cryolesion 2 months after surgery in nineteen cases of pigment fallout

\begin{tabular}{|c|c|c|}
\hline \multirow{2}{*}{ Cryo-lesion } & \multicolumn{2}{|l|}{ Grade } \\
\hline & 1 and 2 & 3 \\
\hline $\begin{array}{l}\text { Patients No. } \\
\text { Per cent. }\end{array}$ & $\begin{array}{r}4 \\
2 \mathrm{I}\end{array}$ & $\begin{array}{l}15 \\
79\end{array}$ \\
\hline
\end{tabular}

Table II Appearance of cryolesion 2 months after surgery in fifty cases of retinal detachment in which pigment fallout or postoperative uveitis did not develop

\begin{tabular}{lll}
\hline & \multicolumn{2}{l}{ Grade } \\
\cline { 2 - 3 } Cryo-lesion & 1 and 2 & 3 \\
\cline { 2 - 3 } & & $\frac{3}{3}$ \\
Patients No. & 47 & 3 \\
Per cent. & 94 & 6
\end{tabular}

The appearance of the treated area 2 months after surgery in the nineteen cases of pigment fallout is shown in Table I. The appearance of the cryotherapy lesion in the separate group of fifty patients studied after retinal detachment surgery who did not suffer either pigment fallout or postoperative uveitis is shown in Table II. The depth of subretinal fluid over the site of cryotherapy and the amount of retina treated in the nineteen cases of pigment fallout and the respective observations for the remaining I4I out of the total of 160 patients are summarized in Table III. The I4I patients not suffering fallout were comparable to the fallout group in age, sex, and duration of detachment.

Table IV shows that fourteen patients had preoperative detachment of the macula; seven suffered pigment fallout at the macula and seven did not. Four patients in whom there had been fallout at the macula showed no postoperative improvement in visual acuity.

\section{Comment}

In assessing the appearance of the treated area 2 months after surgery it was found that 
Table III Depth of subretinal fluid over site of cryotherapy and amount of retina to be treated in nineteen cases of pigment fallout and I4 I normal cases of retinal detachment

\begin{tabular}{|c|c|c|c|c|c|}
\hline \multirow{2}{*}{ Condition } & & \multicolumn{2}{|c|}{ Pigment fallout } & \multicolumn{2}{|c|}{$\begin{array}{l}\text { Retinal } \\
\text { detachment }\end{array}$} \\
\hline & & No. & Per cent. & No. & Per cent. \\
\hline $\begin{array}{l}\text { Depth of } \\
\text { subretinal fluid }\end{array}$ & $\begin{array}{l}\text { Balloon } \\
\text { Moderate } \\
\text { Slight }\end{array}$ & $\begin{array}{r}7 \\
12 \\
0\end{array}$ & $\begin{array}{r}37 \\
63 \\
0\end{array}$ & $\begin{array}{l}43 \\
58 \\
40\end{array}$ & $\begin{array}{l}30 \\
42 \\
28\end{array}$ \\
\hline $\begin{array}{l}\text { Amount of } \\
\text { retina treated }\end{array}$ & $\begin{array}{l}\text { More than } \\
\text { half quadrant } \\
\text { Less than } \\
\text { half quadrant }\end{array}$ & 12 & 63 & 30 & $2 \mathrm{I}$ \\
\hline
\end{tabular}

Table IV Extent of macular involvement in nineteen cases of pigment fallout

\begin{tabular}{llll}
\hline Macula & & No. of cases & $\begin{array}{l}\text { Pigment fallout } \\
\text { at the macula }\end{array}$ \\
Detached & & 7 \\
Flat & 5 & 0 \\
\hline
\end{tabular}

fifteen of the nineteen cases of pigment fallout (79 per cent.) had cryotherapy lesions of Grade 3 severity, at least in part. In the remaining four cases (2 I per cent.) pigmentation of the lesion varied from Grade I to Grade 2. Table II shows that Grade 3 lesions were produced in only three ( 6 per cent.) of the fifty that had not suffered pigment fallout or postoperative uveitis, and the difference between the two groups is significant $\left(\mathrm{P}<0^{\cdot 00} \mathrm{I}\right)$. Thus the production of a Grade 3 lesion may be associated with pigment fallout and represents excessive treatment. It was found that two main clinical factors contributed to the production of over-treatment with cryotherapy-the depth of subretinal fluid at the site of cryotherapy and the amount of retina to be treated.

Subretinal fluid depth at the site of cryotherapy is useful as a guide to the difficulty in detecting the effect of cryotherapy when observed at operation with the indirect ophthalmoscope. Of the nineteen cases of pigment fallout, none had only slight subretinal fluid (Table III). In the remaining I4I cases that did not suffer fallout, forty ( 28 per cent.) had slight subretinal fluid (Table III). This difference is significant $(\mathbf{P}>0.00 \mathrm{I})$. Minimal subretinal fluid facilitates apposition of the detached part of the retina to the pigment epithelium layer by cryoprobe indentation at operation and makes for easy direct observation of the 'end-point' which, provided there is apposition, is taken as the first distinct whiteness of the retina. In this situation over-treatment is less likely to occur. Greater amounts of subretinal fluid make apposition difficult or impossible (Fig. 4) and observation of the endpoint more difficult. If apposition is not possible then the end-point is taken as the subtle blanching of the choroid, seen through the detached part of the retina and subretinal fluid. If distinct whiteness of the detached part of the retina is waited for, then 

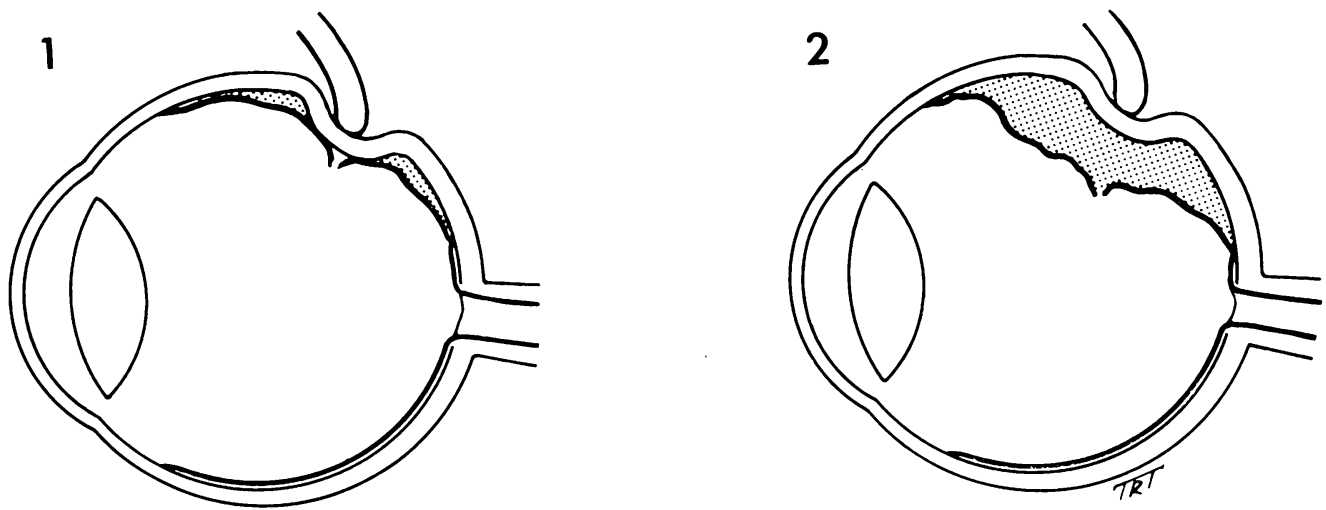

FIG. 4 In the presence of slight subretinal fluid apposition between detached retina and pigment epithelium is possible with cryoprobe indentation (Diagram $\mathrm{I}$ ), whereas with large amounts of subretinal fluid (Diagram 2) apposition is impossible

overfreezing of the pigment epithelium may occur and result in pigment explosion and fallout.

Treatment of large areas of retina results in a greater incidence of pigment fallout. Twelve (63 per cent.) of the patients with fallout had more than half a quadrant treated, whereas in the remaining I4I not suffering fallout only thirty (2 I per cent.) had more than half a quadrant treated; this difference is significant $(\mathrm{P}<\mathrm{O} \cdot 00 \mathrm{I})$. The greater incidence of pigment fallout arising when large areas of the retina are treated may be due to the tendency to freeze the same area of retina more than once. Retinal landmarks (e.g. bloodvessels) may be used to minimize refreezing, in an attempt to separate one application of cryotherapy from the one before, but this is also particularly difficult when there is a considerable depth of subretinal fluid.

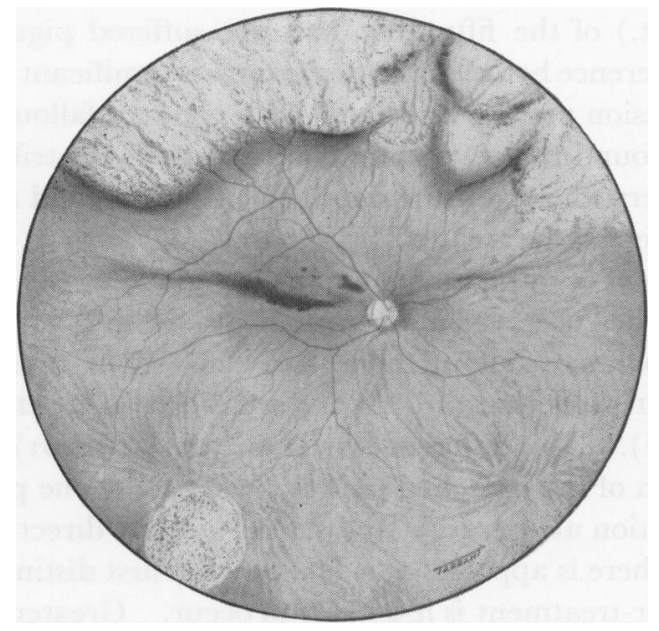

FIG. 5 Pigment fallout which has occurred at the macula after treatment for an upper-half retinal detachment

Pigment fallout at the macula (Fig. 5) occurred in seven of the fourteen cases in which the preoperative detachment involved the macula. This high incidence apparently occurred because the macula is the most dependent part of the eye in the supine patient at operation. 
The extent of recovery of macular function, as expressed in terms of the visual acuity, is always unpredictable when the macula has been detached, but when pigment fallout involves the macula the visual acuity may be further depressed. There was noevidence that fundi which already had evidence of pigment epithelium proliferation (e.g. pigmented lattice degeneration) showed any greater tendency to develop pigment fallout.

POSTOPERATIVE UVEITIS

In this part of the study the sixty cases of retinal detachment treated by local plombage \& and cryotherapy without drainage of subretinal fluid were studied. Seven cases (I 2 per cent.) developed marked postoperative uveitis. Preoperatively six cases had ballooned retinal detachments.

Symptoms and signs

Details of the seven cases are summarised in Table V (overleaf).

All the patients enjoyed an uneventful few days postoperatively with no more than the usual slight discomfort associated with the operative procedure. However, retinal whitening following cryotherapy, usually seen for only the first I or 2 postoperative days, was sustained for 3 or 4 days after operation. On the $5^{\text {th }}$ to 8 th postoperative day the symptoms and signs of a uveitis appeared. Pain was usually quite severe with flare and cells appearing in the anterior chamber. Vitreous haze was found situated over the plomb (Fig. 6) and in two cases ( 2 and 5 ) the haze became generalized.

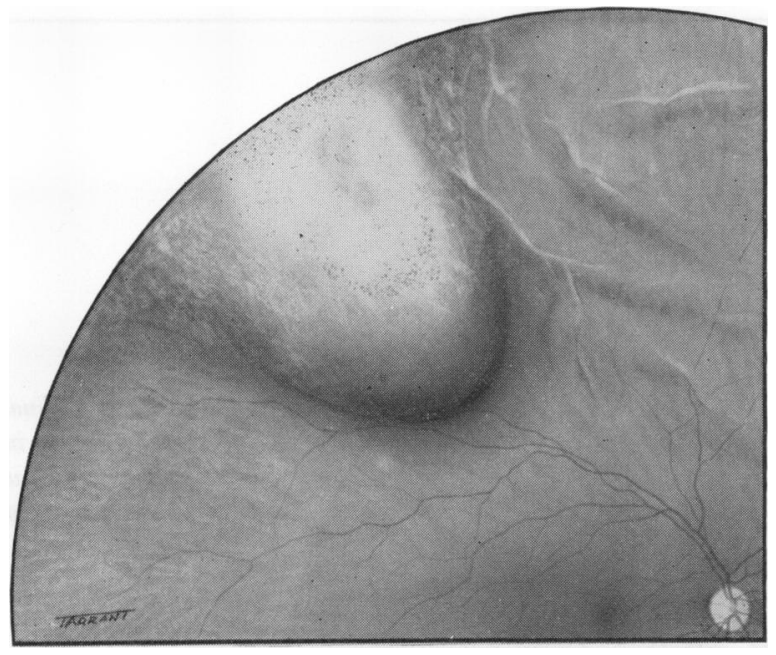

FIG. 6 Vitreous haze overlying radial plomb

Preoperative cultures were sterile in all cases. In four in which a postoperative conjunctival culture was taken no growth was found. In the only case which was explored (Case 5), Staph. aureus (coagulase-positive) was isolated from the plomb which was removed at operation. In this case the underlying sclera was found to be completely normal. In no case was there a mucopurulent discharge.

When these patients were re-examined 2 months after operation all were found to have treated areas corresponding to Grade 3 (Fig. 7, overleaf). 
Table V Details of seven cases of uveitis after cryotherapy

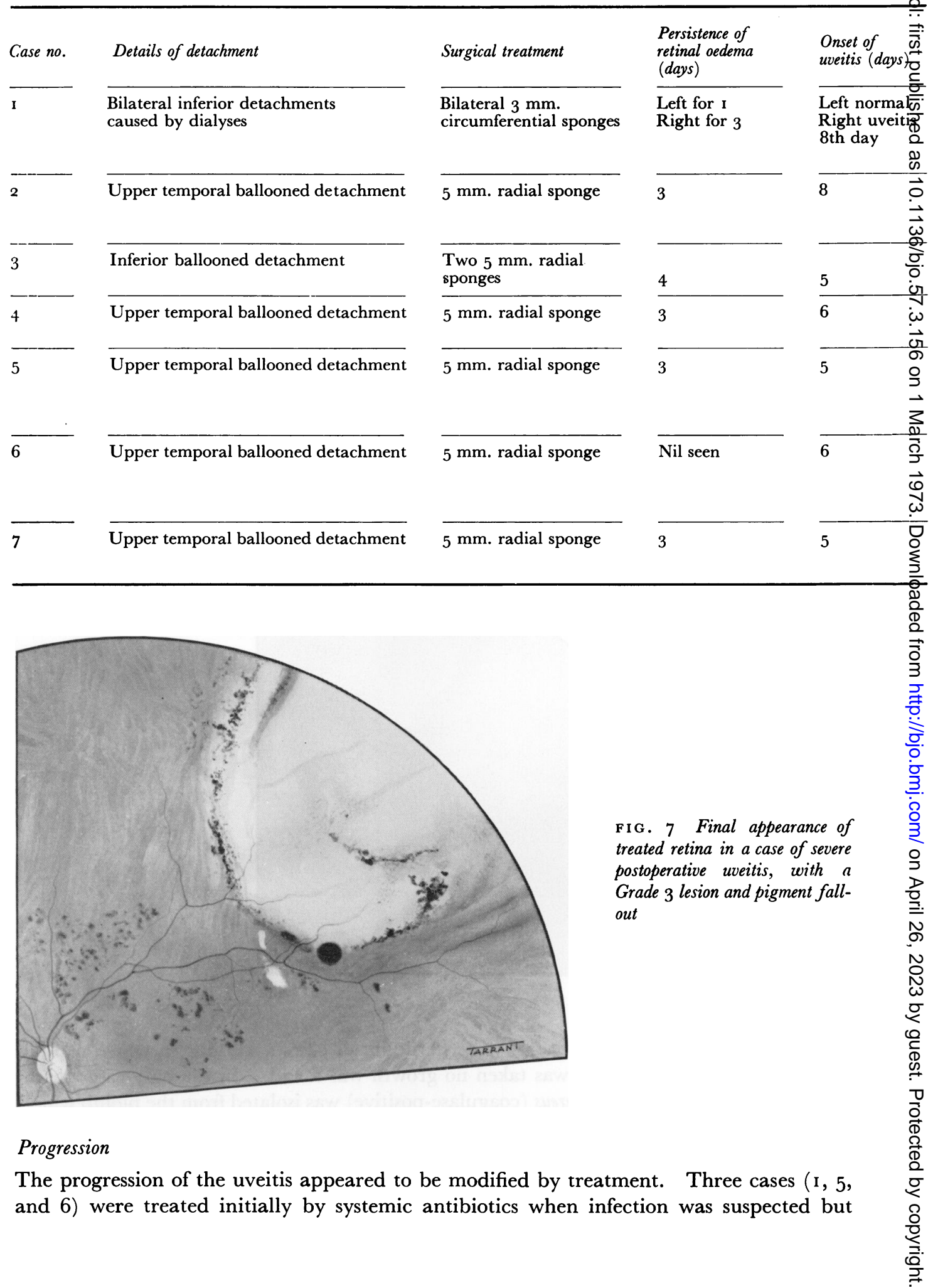


Initial signs of uveitis

Flare and cells

Vitreous haze over plomb

Flare and cells

Vitreous haze over plomb

Flare and cells

Vitreous haze over plomb

Flare and cells

Vitreous haze over plomb

Flare and cells

Generalized vitreous haze

Flare and cells

Vitreous haze over plomb

Flare and cells

Vitreous haze over plomb

\section{Details of treatment}

Systemic ampicillin 2 days

Prednisolone $20 \mathrm{mg}$./day and local steroids added because of increasing uveitis

Immediate improvement

Local steroids for I week

Systemic prednisolone $20 \mathrm{mg}$./day used because of increasing uveitis Rapid resolution

Local steroids only

Local steroids only

Systemic ampicillin and septrin

Marked worsening of symptoms and signs

Plomb removed and prednisolone $20 \mathrm{mg}$./day started

Rapid resolution

Systemic ampicillin and septrin

Worsening

Prednisolone $30 \mathrm{mg}$./day started after 2 weeks

Rapid resolution

Local steroids only

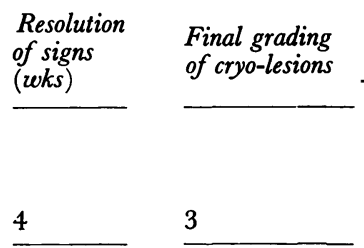

4

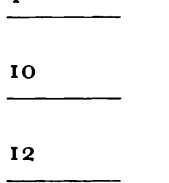

3

3

3

ज

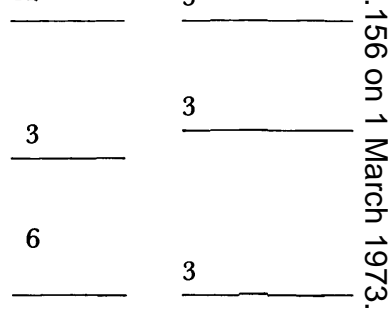

3

8

the symptoms and signs worsened with this treatment; the introduction of systemic $\stackrel{\mathbb{Q}}{\rightrightarrows}$ steroids brought about rapid resolution. Three cases $(3,4$, and 7 ) received only local 9 steroids, which although they improved the anterior uveitis had little effect on the vitreous haze. Case 2 at first received local steroids only but systemic steroids were added after I week and these resulted in rapid resolution of both anterior and posterior uveitis. In 0 the cases treated with both systemic and local steroids (Cases I, 2, 5, and 6), the symptoms and signs had totally resolved 6 weeks from the time of operation. In cases treated with local steroids only (Cases, 3, 4, and 7), resolution took between 8 and i 2 weeks.

\section{Differential diagnosis}

The uveitis produced does not appear to be of infective origin. Scleral abscess, which $\frac{D}{0}$ was associated with diathermy (Lincoff, McLean, and Nano, r965) and resulted in a purulent endophthalmitis, has not been seen since the introduction of cryotherapy. Lincoff, Nadel, and O'Connor (1970) indicated that the infected scleral implant is not an acute condition and although the eye was red and irritable in the postoperative period the earliest time of discovery was 2 weeks. Discharge occurred from the site of the implant and positive conjunctival cultures were usually obtained but signs of intraocular involvement were rarely seen. Treatment consisted of removal of the implant.

A mild transient sterile uveitis is often found after long and complicated detachment procedures; generalized vitreous haze and a few cells in the anterior chamber are found on the first or second postoperative day but the signs usually improve rapidly in the first postoperative week. 


\section{Treatment}

Recognition of the condition by slit-lamp examination and indirect ophthalmoscopy in the postoperative period should lead to prompt treatment with both local and systemic steroids and this will result in rapid resolution of the symptoms and signs. Removal of the implant is not necessary.

\section{Comment}

It seems likely that excessive freezing of the choroid and retina is responsible for the uveitis described. In six out of seven cases there was an unusual amount of retinal oedema after cryotherapy around the retinal tear for the first 3 postoperative days, and all had the appearance of a Grade 3 lesion when assessed 2 months after surgery. In one case, which had bilateral retinal detachments (Case I), one eye, in which recovery was uneventful, showed a lesion of Grades I to 2 while the other, which suffered a marked postoperative uveitis, eventually finished with a Grade 3 lesion. In addition to uveitis, three of the cases seen $(3,6$, and 7 ) had suffered pigment fallout, supporting the probability of excessive cryotherapy. In six cases $(2,3,4,5,6$, and 7$)$ there was a ballooned detachment preoperatively thus increasing the likelihood of overfreezing as already shown (Fig. 4). Early treatment with systemic and local steroids, as well as greatly reducing the postoperative morbidity, brings about more rapid clearing of the vitreous and prevents the risk of permanent vitreous opacity leading to reduced visual acuity or the possibility of fibrotic changes leading to massive vitreous retraction and re-detachment.

\section{Summary}

Pigment fallout and postoperative uveitis associated with the use of cryotherapy in the treatment of retinal detachment was studied, and the final appearance of the treated area was graded to classify the severity of application. In a series of 160 retinal detachments, nineteen (1 2 per cent.) showed pigment fallout. Fifteen of these patients had Grade 3 lesions at least in part when the operative area was assessed 2 months after surgery and in the remaining four the lesions were more evenly pigmented and corresponded to Grades I to 2. The Grade 3 lesion is only occasionally seen (in 6 per cent.) in patients who do not develop fallout or uveitis complications after retinal detachment. It was found that a considerable proportion of the patients with pigment fallout received unnecessarily heavy applications of cryotherapy ( 79 per cent.). It was also found that the two main contributory factors in the production of excessive cryotherapy were deep subretinal fluid at the intended cryotherapy site and a large area of retina needing treatment, causing either overfreezing or refreezing respectively. Pigment fallout at the macula may depress visual acuity but this has yet to be conclusively ascertained.

Postoperative uveitis after external plombage and cryotherapy, without drainage of subretinal fluid, occurred in seven out of sixty cases (1 2 per cent.). The only abnormal physical sign seen in the immediate postoperative period was the persistence of retinal oedema in the treated area. The uveitis which presented 5 to 8 days postoperatively responded well in the cases treated with both local and systemic steroids. In all cases the eventual appearance of the treated area had the characteristics of a Grade 3 lesion. In five of the seven cases there had been large ballooned detachments, resulting in the tendency to overfreeze because of the difficulty in visualizing the cryotherapy reaction at operation, and it is felt that an excessive amount of cryotherapy was responsible for the production 
of this sterile endophthalmitis. The occurrence of pigment fallout in three cases supports the likelihood of overfreezing.

There is no clinical evidence to suggest that the production of a lesion with the characteristics of Grade 3 is necessary to achieve firm adhesion, but such a lesion may be associated with the various complications which have formed the subject of this paper.

Careful observation of the end-point, i.e. choroidal blanching, when there is deep subretinal fluid between the detached part of the retina and the pigment epithelium layer, will help to prevent overfreezing, and a systematic technique using local landmarks will reduce the tendency to refreeze any part unnecessarily when large areas are to be treated.

\section{Conclusion}

Pigment fallout and the type of postoperative uveitis described in this study are due to excessive cryotherapy application and the occurrence of these complications should increase the tendency to conservatism in the application of cryotherapy.

We should like to thank Mr. L. G. Fison and other members of the Retinal Unit, Moorfields Eye Hospital, City Road, for their help and advice, the Audio-Visual Department of the Institute of Ophthalmology, London, for preparing the illustrations, and Miss M. Farrell for invaluable secretarial assistance. This work was in part supported by the Clothworkers' Company. Three illustrations are reproduced by permission of the editorial committee of the Ophthalmological Society of the United Kingdom.

\section{References}

ABrAham, R. K., and SheA, м. (ig68) Trans. ophthal. Soc. U.K., 88, 297

BIETTI, G. (I934) Boll. Oculist., 13, 576

CHIGNell, A. H., Revie, I. H. S., and Glemett, R. S. (I97I) Trans. ophthal. Soc. U.K., 91, 635 CROCK, G. (I967) Ibid., 87, 513

Johnston, G. P., and okun, E. (I969) In "Current Concepts in Ophthalmology", ed. B. Becker and

R. M. Burde, vol. 2, p. io8. Mosby, St. Louis

Lincoff, H. A., Baras, I., and mclean, J. m. (ig65) Arch. Ophthal. (Chicago), 73, i6o

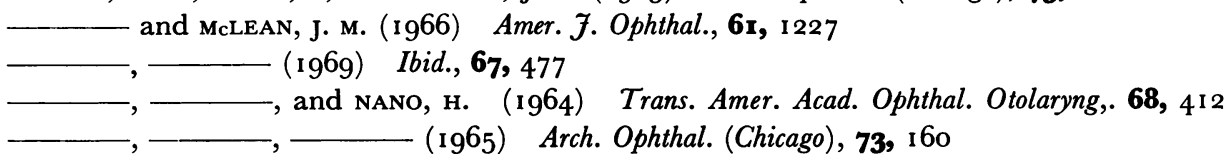

LINCOFF, H. A., NADEL, A., and o'CoNNOR, P. (1970) Ibid., 84, 421

SUDARSKY, R. D., and YANNUZZI, L. A. (1970) Ibid., 83, 395 